\title{
Treatment of victims of trauma
}

\author{
Gwen Adshead
}

The psychiatric sequelae of trauma have been most often discussed in relation to disasters, both natural and man-made, and military conflict. This may sometimes lead psychiatrists to think of posttraumatic stress disorder (PTSD) and other posttraumatic mental illnesses as being somewhat exotic and unusual in general psychiatric practice. In this paper, I propose that PTSD and related disorders are actually common psychiatric problems which require treatment.

The history of the concept will not be reviewed here; interested readers are referred to reviews by Trimble (1985) and Gersons \& Carlier (1992). The problems of adult survivors of childhood trauma will not be discussed in detail here, but there are reasons for thinking that therapeutic approaches based on a PTSD model may sometimes be inappropriate. Concerning the specific problems of war veterans or children, readers are referred to the relevant chapters in textbooks such as Wilson \& Raphael (1993).

\section{The prevalence of post- traumatic disorders}

The prevalence of PTSD and other disorders within a community will depend to some extent on the prevalence of traumatic events in the life of a community. Breslau et al (1991) found a prevalence of traumatic events of $39 \%$. In a heterogeneous community sample in the US, Norris (1992) found a lifetime prevalence of traumatic events of $69 \%$. Most common were bereavements by homicide, suicide or accident; least common was sexual assault.

Studies of prevalence of PTSD in the community have confirmed that PTSD is not a rare diagnosis. Two American studies suggest a lifetime prevalence rate of $1 \%$ for PTSD (Helzer et al, 1987; Breslau et al, 1991). The prevalence of chronic PTSD was $3.45 \%$ (Breslau et al, 1991). There are not yet any equivalent
British studies; however, the British Crime Survey of 1992 (Mayhew et al, 1993) found that over one million physical assaults were reported to the police. Between $500-600$ people a year lose a relative by homicide, and $300-400$ by fatal road traffic accidents. Although the level of criminal violence is lower here than in the US, it is likely that the prevalence of PTSD within the British community may be similar to that of the US. All psychiatric specialities may therefore expect to have to deal with PTSD and related disorders.

\section{Normal responses to trauma}

There is clearly nothing abnormal about feeling bad when bad things happen. It is equally clear that acute psychological stress reactions, however normal, are extremely distressing and uncomfortable. The best analogy is that of the fractured limb; the pain is entirely normal, but may be treated nonetheless. DSM-IV (APA, 1994) and ICD-10 (WHO, 1992) recognise acute stress reactions as diagnostic entities.

The features of acute stress reactions are described in Table 1. It is important to distinguish between normal stress reactions and PTSD. Both diagnoses have symptoms in common; for example, intrusive phenomena such as repetitive thoughts and nightmares. However, by 4-6 weeks, most acute stress reactions will be diminishing or much reduced. It appears that the majority of people surviving a traumatic event will make a spontaneous, if painful recovery. Only a minority will go on to develop PTSD and related disorders (about 25-40\%; Green et al, 1990; Green, 1993). However, the subjective unpleasantness of acute stress reactions should not be underestimated, and clinicians may need to remind families of this. Recovery is the norm, but may be delayed where there is further stress.

Dr Gwen Adshead is Lecturer in Forensic Psychiatry, Institute of Psychiatry, De Crespigny Park, Denmark Hill, London SE5 8AF. She trained in forensic psychiatry at St George's Hospital, and has worked as a therapist with both perpetrators and survivors of trauma. 


\section{lable. I. Vinmil}

Time period

Anticipation phase

(often not present)

Immediate

Short-term (1-6 weeks)

Long-term

(6 weeks to 6 months)
Effects

Anticipatory anxiety/fear; denial

Shock, numbness, disbelief. Acute distress. Dissociation and denial

High levels of arousal. Intrusive phenomena of trauma: thoughts, flashbacks, nightmares. Poor concentration. Disturbed sleep, appetite, libido. Irritability. Persistent fear and anxiety, especially when reminded of trauma: leads to avoidance behaviour

Features described above persist, but should decrease in intensity and frequency. Increased avoidance behaviour. Irritability is often most persistent. Substance abuse is common as a way of managing arousal

NB. DSM-IV criteria for acute stress disorder specify symptoms as not persisting for more than four weeks.

\section{Post-traumatic stress disorder}

Table 2 outlines the DSM-IV criteria for PTSD. The phrase "outside the range of usual human experience" has now been dropped from the definition. This is in part the result of research which suggests that the perception of fear and threat is crucial in the genesis of PTSD, so that PTSD is possible after events which are common but terrifying, such as road traffic accidents, or domestic violence (Walker, 1984; Mayou et al, 1993).

\section{Risk factors for PTSD}

Table 3 shows some of the risk factors for PTSD. Several studies have confirmed that the magnitude and degree of exposure to the stressor influences the risk of developing PTSD. Previous personality traits, coping styles and experiences also influence the development of PTSD (McFarlane, 1988). There is evidence that those individuals with a pre-trauma psychiatric history are at increased risk of developing PTSD (Smith et al, 1990). Recent studies of

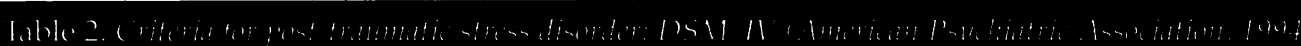

Criterion

A. The stressor criterion

B. Intrusive phenomena

C. Avoidance phenomena

D. Hyperarousal phenomena

E. Duration

F. Impairment of social function

\section{Symptoms required to fulfil criterion}

Involves objective and subjective elements:

(i) the person has been exposed to an event involving actual or threatened death, or serious injury, or threat to physical integrity of self or others

(ii) the person's response involved intense fear, helplessness or horror

Persistent re-experiencing in at least one of five ways: intrusive recollections, dreams, flashbacks, psychological distress or physiological reaction when exposed to reminders of the trauma

At least 3 of 7 ways of avoidance behaviour: e.g. avoidance of thoughts or feelings about trauma, amnesia, decreased interest, feelings of detachment

At least 2 of 5 possible symptoms: e.g. insomnia, irritability, poor concentration

At least one month

The symptoms must cause clinically significant distress or social dysfunction

NB. Acute PTSD < 3 months duration; chronic PTSD > 3 months duration. 
soldiers suggest that those with histories of abuse in childhood are at increased risk of developing PTSD (Bremner et al, 1992). Although the adult sequelae of childhood trauma cannot be discussed here at any length, it is relevant to note that some workers have suggested that these problems may be conceptualised as chronic and 'complex' PTSD (Herman, 1992). In relation to crime, a previous history of victimisation increases the risk of developing PTSD. Once again, this suggests a complex interaction of constitutional responses to arousal, cognitive coping style and experience of new trauma.

The development of PTSD is therefore the complex result of the interaction of many factors. A clinical example seen by the author makes the point. Five men were involved in a helicopter crash. Superficially, all were exposed to severe lifethreatening trauma, involving grotesque imagery (a fellow passenger was decapitated and his mutilated remains spread over the crash site). On the basis of the nature of the trauma alone, one might expect all the survivors to develop PTSD. However, at 18 months post-trauma, only two of the five had failed to make a reasonable recovery after a normal stress response. Only one man developed PTSD. His subjective experience of the trauma was particularly unpleasant; there was also evidence that his pre-trauma personality put him at risk. The other man who failed to recover did not have PTSD, but instead was chronically anxious in a way that severely affected his work performance. Each man's subjective account of the crash was different to the others, making the point that subjective experience interacts with objective severity to influence the development of psychopathology. It is therefore not possible to state that only extremes of trauma, or individual psychological vulnerability, lead to the development of
PTSD. The relative contributions of both individual past and the traumatic experience need to be considered during assessment, and have implications for treatment choice.

\section{Psychopathology and pathophysiology of PTSD}

One of the principal debates about PTSD relates to whether PTSD is seen as an anxiety disorder or a mood disorder (Davidson \& Foa, 1991). Other possible diagnostic categories are dissociative disorders, or a disorder of personality. The rationale for the various positions is detailed in Box 1 .

Category definition has implications for treatment. Most treatment for PTSD is currently symptomatically based. The pathophysiology of PTSD remains relatively unclear, with research findings suggesting the involvement of the hypothalamicpituitary axis, central monoamine regulation and endogenous opioids. In addition to the neurophysiological disturbances exhibited by patients with PTSD, there is also evidence of cognitive psychopathology, e.g. subjects with PTSD are more sensitive to percepts indicating threat, and respond more vigorously to such cues (Cassiday et al, 1992).

\section{Other post-traumatic problems}

It cannot be over-emphasised that PTSD is not the only problem that survivors of trauma face. 'Pure' PTSD after trauma is comparatively rare, and

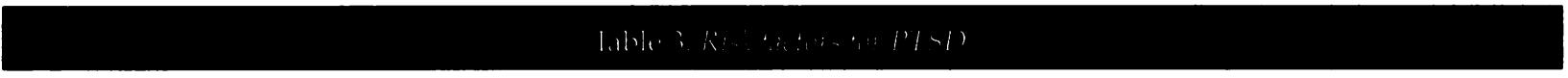

Aspect of trauma

The stressor itself

Experience during the trauma

The individual subject
Risk factors

Duration and magnitude of exposure to the stressor. Stressors which: are sudden and/or occur with no warning; cause multiple loss of life; mutilate bodies or give rise to grotesque imagery; are caused by criminal violence, especially sexual

When subject perceived life to be at real risk: "I thought I was going to die". Perceived uncontrollability of events, and helplessness. Perception of grotesque imagery: especially of human remains, or of children. Witnessing or carrying out atrocities, e.g. murder, torture

Previous psychiatric illness. Previous neuroticism. Previous exposure to trauma; especially childhood trauma. Previous coping style. Denial of trauma and/or avoidance

Denial of trauma by others or dismissal of experience:"not being believed" 
Box 1. Models of psychopathology in PTSD

\section{(1) An anxiety disorder?}

PTSD sufferers experience fear, anxiety and avoidance behaviour (like phobics).

Intrusive phenomena resemble obsessive-compulsive disorder phenomena.

Introversion and neuroticism are common personality traits in both PTSD and anxious populations.

Some patients improve with exposure therapy.

(2) A mood disorder?

Patients with PTSD experience sadness and grief, independent of bereavement.

Comorbidity with depression is very common.

Vegetative symptoms are very similar: loss of sleep, appetite, libido.

Both groups experience avoidance, numbing and loss of interest.

(3) A dissociative disorder?

Flashbacks and amnesia are common in patients with dissociative disorder and PTSD.

(4) A personality disorder?

Considerable overlap of symptoms of PTSD with symptoms of borderline personality disorder. Some overlap of symptoms with those of antisocial personality disorder (e.g. antisocial behaviour, irritability).

Some evidence that trauma can induce personality change.

(5) A separate neurophysiological disorder?

Effects such as low MAO activity, increased excretion of urinary beta-endorphin, therapeutic response to both serotonergic drugs, and drugs that affect the locus coeruleus.

comorbidity is the norm. Depression is the commonest co-diagnosis, and may be the commonest posttraumatic disorder overall. Other post-traumatic psychiatric illnesses include anxiety disorders, such as panic disorder or phobic disorders. Full-blown PTSD is relatively uncommon, and partial PTSD may be more likely, particularly in a chronic form. Substance abuse may be the primary presenting problem, masking intrusive symptoms of PTSD. Marked changes in personality, in terms of personal interaction with others, may cause more problems than any of the other post-traumatic disorders, especially when this is accompanied by substance abuse or violent behaviour (Southwick et al, 1993).

As in general psychiatric practice, it is important to consider the influence of the patient's illness on other areas of his life, in relation to its effect on the process of recovery and the prognosis. The symptoms of many of the post-traumatic disorders may be troublesome for families and employers, particularly in the first 6-12 months after trauma. Patients may be chronically irritable and withdrawn for weeks on end, in a way which is alien to them and their families. Work performance deteriorates, but patients often find it impossible to discuss the reasons for this with their employers, who may not be sympathetic anyway. This is a particular problem when the trauma occurred at work (and compensation is being claimed), or where there is a work culture of denial of distress. Examples of this are the emergency or public services, and health care professionals. Even though the 'macho' culture is changing to some degree, especially within the emergency services, the process is slow, and patients may encounter hostility and rejection by their workplaces.

Families, friends and employers are usually unfamiliar with the time-scale of normal recovery, let alone post-traumatic psychiatric illness. Most people do not realise that normal recovery may take six months, or longer if there are further stressors, and may become impatient with survivors who may be seen as being difficult or weak. Local social support often reduces as press reports of the trauma diminish, and it may seem, to health care professionals or family members, as though the trauma happened a long time ago. The pathology of PTSD, however, makes the trauma continually real to the survivor (Horowitz, 1973).

Many major personal disasters are never reported in the press at all. In peacetime, and between major disasters, the principal cause of traumatic stress responses is crime, of which the impact on the victim is rarely reported unless it is fatal (Kilpatrick et al, 
1989). This applies to both men and women alike (Stanko \& Hobdell, 1993). The best example of this is the plight of the families of murder victims. The killer is often a member of the family, and so relatives must cope with multiple losses. In one case known to the author, a woman presented after her husband murdered their daughter. The killing took place to prevent the daughter from telling her mother about her father's 20-year affair with a family friend. This woman lost not only her daughter and her husband, she also lost her experience of her marriage and the support of a trusted friend. She was also without funds as the husband had been the principal earner, and she did not have access to the bank account. The trial did not take place for a year, and the funeral was delayed several times for post-mortem reports for both defence and prosecution. Other problems of families of murder victims are well described by other authors (Parkes, 1992; Black et al, 1993).

These social and legal aspects of post-traumatic dysfunction have a profound influence on the management and prognosis of PTSD, and can cause major setbacks in treatment. A man who becomes homeless because of domestic violence related to his post-traumatic irritability may be unable to cooperate, or tolerate a treatment programme which is quite taxing. In-patient treatment may be indicated for such patients. Patients with PTSD as a result of crime have particular problems. Not only may they be involved with reminders of the stressor, such as police identification parades or court appearances, they may be at continued risk of further trauma, such as threats from the defendant.

\section{Assessment}

The need for thorough assessment is particularly pressing for the post-traumatic disorders. It may not always be immediately apparent that the presenting complaint is post-traumatic in origin, and it is important to bear this possibility in mind. This is particularly so for victims of crime, especially sexual assault and domestic violence, who may find it difficult to tell others about their experiences, and who may not define their experience as a crime. There is some evidence that health care professionals do not ask enough about trauma, and there is also evidence that patients do not resent this (Friedman et al, 1992). Patients suffering posttraumatic disorders may find it difficult to describe spontaneously their experiences, and appreciate a tactful enquiry. In addition, patients are frequently not referred to general practitioners (GPs) until some time after traumatic events, so that the importance of an event that may have taken place a year before may be overlooked.

Having enquired about trauma, the assessor must be prepared to hear the patient's account. This entails making enough time for the patient to do this comfortably. If a history of a trauma is already known at referral, then it may be helpful to suggest to patients that history-taking takes place in two stages: firstly, a general psychiatric history, and secondly, an account of the trauma and post-traumatic events. This gives the patients some warning, and allows them to prepare themselves, reducing anticipatory anxiety. If the trauma is disclosed de novo, and the patient wants to give an account of his experience, then it is important to allow this to take place. Patients with post-traumatic disorders are very sensitive to the understandable reluctance of others to hear their stories. It may be necessary to let the patient talk for a little time, then negotiate a time for the patient to return and for the assessment to continue.

What is not advisable is dismissing patients' disclosures of trauma. There is no evidence that patients benefit from being told to 'forget all about it' or 'put it out of your mind'. Intrusive phenomena which cannot be voluntarily excluded are characteristic of PTSD; patients are not actually capable of 'just forgetting'. Dismissal also gives a message to the patient that the health care professional does not want to hear, or does not believe what he is being told. Even in cases where the professional needs to maintain a true scepticism about the patient's account of events (such as in medico-legal work), this does not entail an unsympathetic manner, which in any case will impede the assessment.

Apart from tactfully and sensitively facilitating the patient's account of trauma, psychiatric assessment proceeds along usual lines. Interviews with family members may be invaluable for an impression of the pre-traumatic state of the patient, and may also give an insight into the course of the posttraumatic sequelae. Discussion with the GP and examination of the records may yield more valuable information about the patient's pre-traumatic state.

Key questions in the assessment are outlined in Box 2. If the trauma happened only recently (say in the last 3-6 months), then some spontaneous progress may still be made, or augmented with support. Positive signs may be the diminishing frequency of nightmares, decreasing use of alcohol, and the return of appetite. Spontaneous progress may be retarded by degree of trauma, simultaneous losses and physical ill-health; patients may not begin the process of psychological recovery until their physical state allows. If they are making progress, then it may be that all they need is pharmacological support for the remaining symptoms, information about the natural course of stress reactions and advice from the GP. The support of community 
Box 2. Key questions in the assessment of post-traumatic disorders

\section{When did the trauma occur?}

How long has the person had symptoms?

How have they coped to date?

What resources do they have to support them?

Is there a previous history of trauma?

Is there a pre-traumatic history of psychiatric illness?

Was the trauma they suffered a 'risky' one? (e.g. criminal assaults of grotesque imagery)

psychiatric nurses may be useful here.

GPs are obvious professionals to be involved in the management of stress reactions. If patients are referred to psychiatry while still in the normal phase of response, these possibilities may be considered:

(1) The patient's GP may lack information about PTSD, and may need information and consultation with the psychiatrist.

(2) The GP/practice counsellor/psychologist has particular concerns about the patient, because of the nature of his/her symptoms.

(3) The GP or practice team may not be able to provide the time needed for support.

(4) The nature of the trauma is particularly serious.

A case history may act as an example. Miss A. was abducted on the street by strangers, and gangraped in the back of a van for several hours before being released. She was reluctant to talk to her GP who was very concerned. Her family were distraught. She was referred to a general psychiatrist as an outpatient, for support and counselling.

It is important to enquire about any previous history of trauma. A previous psychiatric history may make the development of PTSD more likely. If the trauma is a criminal one, it is important to ascertain the state of any legal proceedings, since this will impact on treatment and progress.

\section{Indications for treatment}

Treatment approaches for the post-traumatic disorders need to be comprehensive, flexible and geared to what the patient can tolerate. The principal treatment modalities are :
(1) behavioural and cognitive strategies (Foa \& Rothbaum, 1989)

(2) short- and long-term psychological therapies

(3) medication (Davidson, 1992).

All three modalities may form part of different therapeutic strategies for the same patient over time, depending on the patient's needs (Southwick \& Yehuda, 1993). Table 4 shows the range of treatments available, the optimal types of therapy for different disorders, and their timing.

The rationale for some behavioural and cognitive treatments is the breaking of the cycle of intrusion and avoidance described in Horowitz's model of PTSD. By exposing the patient to their feared memories, or their thoughts about the trauma, avoidance is reduced, and control over intrusion is introduced. Different types of exposure therapy include imaginal flooding, systematic desensitisation and exposure to the feared stimulus in both imagination (using tapes) and in vivo. Exposure therapy may be the first line of treatment, or may follow treatment for other post-traumatic disorders such as depression. Concurrent prescription of antidepressants in PTSD may be necessary to increase the patient's ability to tolerate the exposure programme. It is likely that exposure to feared memories is an important part of most posttraumatic therapies (Richards \& Rose, 1991).

The other rationale for cognitive and behaviour therapies is based on PTSD as a failure of cognitive processing (Foa et al, 1989). Cognitive therapies have been shown to be effective for some types of trauma (Foa et al, 1991; Resick \& Schnike, 1992). A more recent development in cognitive processing techniques using saccadic eye movement has been described by Shapiro (1989) and Page \& Crino (1993). This technique remains controversial, and its relationship to other treatment modalities uncertain. Behavioural and cognitive strategies are probably indicated as first line treatments where there is good psychological health before the traumatic event, and where the event itself is discrete.

Brief and longer-term psychodynamic psychotherapy, both group and individual, may be indicated where there is prior history of trauma, psychological vulnerability, or where the trauma is the result of personal victimisation (Lindy et al, 1983). Group psychotherapy may be of particular use where the trauma occurs in a group context, such as occupational settings, or transport disasters. Therapeutic communities have been used principally in veteran populations (Silver, 1986). Brief group work is possible when the group focuses on a particular task, such as the Critical Incident Stress Debriefing model described by Mitchell (1983). Group work may be of particular use after sexual 


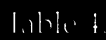

Type of disorder

Acute stress responses

PTSD (acute)

PTSD (chronic)
Indicated treatments

Debriefing. Social supports. Pharmacological supports, e.g. hypnotics. Information and advice to families

If intrusive phenomena prominent: exposure therapy may be the first line of treatment. In addition/sequence: cognitive therapy; brief psychodynamic psychotherapy; antidepressants (especially where avoidance prominent)

Cognitive-behavioural approaches may still be effective: group or individual. Long-term psychotherapy: group or individual. Antidepressants/lithium. Carbamazepine. May be worth trying exposure therapy if trauma was never discussed before. assaults, where by making the experience less individual, shame and guilt may be reduced (Roth et al, 1988).

Individual psychodynamic approaches may focus on restoring defences, and helping the patient to think about those events which appear to have no meaning (Garland, 1991). Short-term (12-week) packages of individual therapy have been described by Marmar (1991) and may be especially useful for a discrete event such as a traumatic bereavement. Such brief approaches are not indicated where there is extensive previous trauma or severe concurrent psychopathology. In such cases, where a traumatic experience resonates with prior losses or childhood trauma, long-term individual therapy may be indicated.

Medication has an important role in the treatment of post-traumatic disorders, both as symptomatic relief, but also as directly addressing pathology. A detailed account of the use of various types of medication is given in Davidson (1992). Antidepressants, especially the serotonergic agents, may be helpful, as may tricyclics because of their hypnotic effects. Medication alone is unlikely to be helpful, but may be necessary to enable patients to undertake other types of therapy later.

Once a diagnosis of PTSD has been made, treatment should be vigorous, because chronic PTSD is hard to treat. Even if the traumatic events took place some time before ( $>1$ year), it may still be worth attempting exposure therapy, if the patient can tolerate it. Antidepressant treatment should also be instituted. However, the prognosis is not good. Although the natural history of the disorder is of very gradual improvement over time, the concurrent effects on family and work life continually retard this process. Once chronic PTSD is established, the therapeutic focus may need to be these concurrent problems (Hammarberg \& Silver, 1994).

There are particular questions relevant to the selection of treatment:
(1) What is the worst problem at the moment?

If intrusive phenomena are prominent, this may suggest exposure therapy as part of a cognitivebehavioural package. If depression and distress are worst, then regular supportive therapy sessions plus antidepressants may be most effective.

(2) What supports does this person have?

Many forms of treatment for PTSD are quite stressful. It is therefore important to ensure that the patient will be well supported, and that the family supports are informed about the nature and process of therapy.

(3) What solutions to stress is the patient adopting now?

If a patient is abusing alcohol or drugs as a means of managing his/her PTSD symptoms, then this problem needs to be addressed before any specific PTSD treatment can be implemented. Rarely, patients may present with acts of deliberate selfharm, such as overdoses, and these should not be dismissed as 'attention-seeking'.

\section{Efficacy of treatment}

There is a lack of information about the efficacy of some types of treatment for PTSD. Relevant studies have been conducted by Foa et al (1991), Richards \& Rose (1991), Brom et al (1989) and others. Methodological problems include obtaining homogeneous samples, and ethical concerns about the use of control subjects. It appears that treatment for 'pure' PTSD can be efficacious, but that there is enormous variation in the clinical presentation, and 
the comorbidity complicates treatment. In subjects with good pre-traumatic psychological health, and where there is a discrete traumatic event, cognitivebehavioural strategies can be very effective. In more complex cases, individual psychotherapy may need to be added, along with appropriate medication. Those patients who are depressed may not be suitable for exposure therapy until depression has lifted.

There is a lack of information about the efficacy of treatment for victims of non-sexual crime and smaller disasters, such as road traffic accidents. There is also a lack of information about the largescale efficacy of all the psychological treatments. Some treatments, such as cognitive-behavioural therapies, have been tested in controlled conditions; others have only been evaluated on a case-by-case basis. One particular intervention which still requires evaluation is the stress debriefing model, advocated for the prevention of PTSD in survivors of trauma. Debriefing is offered in the initial posttraumatic stage (1-3 weeks), and is now standard procedure in some work places, although its efficacy in preventing the development of PTSD is still uncertain. However, there is evidence that those who have debriefing find it helpful (Robinson \& Mitchell, 1993).

\section{Conclusions}

Traumatic events are not uncommon in civilian life, especially as a result of crime. Survivors of traumata, both large and small scale, are likely to appear in general and specialist psychiatric clinics requiring treatment, and there is evidence to suggest that treatment for the post-traumatic disorders can be very effective. There is no evidence to suggest that the majority of patients who present with posttraumatic psychopathology are manufacturing those symptoms for the purposes of compensation. Although this view lingers rigidly on in the minds of some psychiatrists, 30 years of research suggest that there really are post-traumatic disorders which need and respond to treatment.

\section{Helpful agencies}

National Association of Victim Support Schemes 01717359166

Compassionate Friends ( help for families of murder victims) 01272539639

Aftermath ( help for families of offenders) 01742326166

Cruse ( help for the bereaved)

01714317122
Medical Foundation for the Victims of Torture 01714820219

Royal Free Clinic (for traumatised children) 01718302049

\section{References}

American Psychiatric Association (1994) Diagnostic and Statistical Manual of Mental Disorders ( 4th edn) (DSM-IV). Washington, DC: American Psychiatric Press.

Black, D., Harris-Hendriks, J. \& Kaplan, A. (1993) When Father Kills Mother. London: Routledge.

Bremner, J., Southwick, S., Johnson, D.,et al (1992) Child physical abuse and combat-related post-traumatic stress disorder. American Journal of Psychiatry, 150, 235-239.

Breslau, N., Davis, G., Andreski, P., et al (1991) Traumatic events and PTSD in an urban population of young males. Archives of General Psychiatry, 48, 216-222.

Brom, D., Kleber, R. J. \& Defares, P. (1989) Brief psychotherapy for post-traumatic stress disorders. Journal of Consulting and Clinical Psychology, 57, 607-612.

Cassiday, L., McNally, R. \& Zeitlin, S. (1992) Cognitive processing of trauma cues in rape victims. Cognitive Research and Therapy, $16,283-295$.

Davidson, J. (1992) Review of the pharmacological treatments for PTSD. British Journal of Psychiatry, 160, 309-314.

— \& Foa, E. (1991) Diagnostic issues in PTSD: Considerations for the DSM-IV. Journal of Abnormal Psychology, 100, 346-355.

— \& (1993) PTSD: DSM-IV and Beyond. Washington, DC: American Psychiatric Press.

Foa, E. \& Rothbaum, B. (1989) Behavioural psychotherapy for PTSD. International Review of Psychiatry, 1, 219-226.

-, Steketee, G. \& Rothbaum, B. (1989) Behavioural-cognitive conceptualisation of PTSD. Behaviour Therapy, 20, 155-176.

-, Rothbaum, B., Riggs, D., et al (1991) Treatment of PTSD in rape victims: a comparison between cognitive-behavioural procedures and counselling. Journal of Consulting and Clinical Psychology, 59, 715-723.

Friedman, L. S., Samet, J. H., Roberts, M. S., et al (1992) Inquiry about victimisation experiences. Archives of Internal Medicine, 152, 1186-1190.

Garland, C. (1991) External disasters and the internal world: an approach to the psychotherapeutic understanding of survivors. In Textbook of Psychotherapy in Psychiatric Practice (ed. J. Holmes). London: Churchill Livingstone.

Gersons, B. \& Carlier, I. (1992) PTSD: The history of a recent concept. British Journal of Psychiatry, 161, 742-749.

Green, B. (1993) Identifying survivors at risk. In International Handbook of Traumatic Stress Syndromes (eds J. Wilson \& B. Raphael). Washington, DC: Plenum.

- Lindy, J., Grace, M., et al (1990) Buffalo Creek survivors in the second decade. American Journal of Orthopsychiatry, 60, 43-54.

Hammarberg, M. \& Silver, S. (1994) Outcome of treatment for PTSD in a primary care unit serving Vietnam veterans. Journal of Traumatic Stress, 7, 195-216.

Helzer, J., Robins, L. \& McEvoy, L. (1987) PTSD in the general population. New England Journal of Medicine, 317, 1630-1634.

Herman, J. (1992) Complex PTSD: a syndrome in survivors of prolonged and repeated trauma. Journal of Traumatic Stress, 5, 377-391.

Horowitz, M. (1973) Phase oriented treatment of stress response syndromes. American Journal of Psychotherapy, 27, 506-515.

Kilpatrick, D., Saunders, B. E. \& Amick McMullen, A. (1989) Victim and crime factors associated with the development of crime related PTSD. Behaviour Therapy, 20, 199-214.

Lindy, J. D., Green, B., Grace, M., et al (1983) Psychotherapy with survivors of the Beverley Hills Supper Club. American Journal of Psychotherapy, 4, 593-610.

McFarlane, A. (1988) The longitudinal course of PTSD: the range of outcomes and predictors. Journal of Neroous and Mental Disease, 176, 30-39. 
Marmar, C. (1991) Brief dynamic psychotherapy of PTSD. Psychiatric Annals, 21, 405-414.

Mayhew, P., Maung, N. A. \& Mirrlees-Black, C. (1993) The 1992 British Crime Survey. London: HMSO.

Mayou, R., Bryant, B. \& Duthie, R. (1993) Psychiatric consequences of road traffic accidents. British Medical Journal, 307, $647-651$.

Mitchell, J. (1983) When disaster strikes ... the Critical Incident Stress Debriefing process. Journal of Emergency Medical Services, 8, 36-38.

Norris, F. (1992) Epidemiology of trauma: frequency and impact of different potentially traumatic events on different demographic groups. Journal of Consulting and Clinical Psychology, 60, 409-418.

Page, A. \& Crino, R. (1993) Eye movement desensitisation: a simple treatment for PTSD? Australia and New Zealand Journal of Psychiatry, 27, 288-293.

Parkes, C. (1992) Psychiatric problems following bereavement by murder or manslaughter. British Journal of Psychiatry, 162, 49-54.

Resick, P. \& Schnike, M. (1992) Cognitive processing therapy for sexual assault victims. Journal of Consulting and Clinical Psychology, 60, 748-756.

Richards, D. \& Rose, J. (1991) Therapy for PTSD: four case studies. British Journal of Psychiatry, 158, 836-840.

Robinson, R. \& Mitchell, J. (1993) Evaluation of psychological debriefings. Journal of Traumatic Stress, 6, 367-382.

Roth, S., Dye, E. \& Liebowitz, V. (1988) Group therapy for sexual assault victims. Psychotherapy, 25, 82-93.

Shapiro, F. (1989) Efficacy of eye movement desensitisation procedure in the treatment of traumatic memories. Journal of Traumatic Stress, 2, 199-223.

Silver, S. M. (1986) An inpatient program for PTSD: context as treatment. In Trauma and its Wake (vol. 2) (ed. C. Figley). New York: Brunner-Mazel.

Southwick, S. \& Yehuda, R. (1993) The interaction between pharmacotherapy and psychotherapy in the treatment of PTSD. American Journal of Psychotherapy, 47, 404-410.

- \& Giller, E. (1993) Personality disorders in treatment seeking combat veterans with PTSD. American Journal of Psychiatry, 150, 1020-1023.

Smith, E., North, C., McCool, R., et al (1990) Acute post-disaster psychiatric disorders: identification of persons at risk. American Journal of Psychiatry, 147, 202-206.

Stanko, E. \& Hobdell, K. (1993) Assault on men. British Journal of Criminology, 33, 400-415.

Trimble, M. (1985) Post-traumatic stress disorder: history of a concept. In Trauma and its Wake (vol. 1) (ed. C. Figley). New York: Brunner-Mazel.

Walker, L. (1984) Battered Woman Syndrome. New York: Springer. Wilson, J. \& Raphael, B. (eds) (1993) International Handbook of Traumatic Stress Syndromes. New York: Plenum.

World Health Organization (1992) The ICD-10 Classification of Mental and Behavioural Disorders. Geneva: WHO.

\section{Multiple choice questions}

1 Normal stress reactions:

a have similar symptoms to PTSD

b normally resolve spontaneously

c do not persist beyond three months

d may persist for up to 12 months

e may be aggravated by other stressors
2 PTSD:

a only occurs after large scale disasters

b occurs in all those who survive disasters

c does not occur after criminal assault

d occurs in $20-40 \%$ of survivors

e has the same lifetime prevalence as schizophrenia

3 The risk of developing PTSD:

a increases with the magnitude of exposure to the stressor

b is not affected by the patient's premorbid personality

c is affected by patients' previous experience of trauma

$\mathrm{d}$ is increased if there is a pretraumatic history of psychiatric illlness

e may be reduced by early intervention

4 Assessment of PTSD:

a It is damaging to enquire about histories of trauma

b Patients should be advised to "put it behind them"

c Family members may provide valuable information

d Comorbidity is the norm

e Depression and substance abuse are common coexisting disorders

5 Treatment of PTSD:

a Medication alone will provide symptomatic relief

b All psychological therapies emphasise recalling the trauma

c Exposure therapy is the first line of treatment after most kinds of trauma

d Exposure therapy may be effective for many years after the trauma

e PTSD responds poorly to treatment

\section{MCQ answers}

\begin{tabular}{llllllllll}
$\mathbf{1}$ & & $\mathbf{2}$ & & $\mathbf{3}$ & & $\mathbf{4}$ & & $\mathbf{5}$ & \\
$\mathbf{a}$ & $\mathbf{T}$ & $\mathbf{a}$ & $\mathbf{F}$ & $\mathbf{a}$ & $\mathbf{T}$ & $\mathbf{a}$ & $\mathbf{T}$ & $\mathbf{a}$ & $\mathbf{F}$ \\
$\mathbf{b}$ & $\mathbf{T}$ & $\mathbf{b}$ & $\mathbf{F}$ & $\mathbf{b}$ & $\mathbf{F}$ & $\mathbf{b}$ & $\mathbf{F}$ & $\mathbf{b}$ & $\mathbf{F}$ \\
$\mathbf{c}$ & $\mathbf{F}$ & $\mathbf{c}$ & $\mathbf{F}$ & $\mathbf{c}$ & $\mathbf{T}$ & $\mathbf{c}$ & $\mathbf{T}$ & $\mathbf{c}$ & $\mathbf{T}$ \\
$\mathbf{d}$ & $\mathbf{T}$ & $\mathbf{d}$ & $\mathbf{T}$ & $\mathbf{d}$ & $\mathbf{T}$ & $\mathbf{d}$ & $\mathbf{T}$ & $\mathbf{d}$ & $\mathbf{T}$ \\
$\mathbf{e}$ & $\mathbf{T}$ & $\mathbf{e}$ & $\mathbf{T}$ & $\mathbf{e}$ & $\mathbf{T}$ & $\mathbf{e}$ & $\mathbf{T}$ & $\mathbf{e}$ & $\mathbf{F}$ \\
\hline
\end{tabular}

